\title{
LIGHTNING \\ ABOVE THE CLOUDS
}

* Alejandro Luque ${ }^{1}$ and Ute Ebert ${ }^{2,3}$

* ${ }^{1}$ Instituto de Astrofísica de Andalucía, IAA-CSIC, Granada, Spain

* ${ }^{2}$ Centrum Wiskunde \& Informatica (CWI), Amsterdam Science Park, The Netherlands

* ${ }^{3}$ Eindhoven University of Technology, The Netherlands

DOI: 10.1051/epn/2010501

The centuries-old quest to understand lightning received an unexpected boost 20 years ago. While testing a camera for a sounding rocket, a group of scientists recorded a huge electrical discharge tens of kilometers above a thunderstorm. This observation spawned a fertile area of research on transient luminous events in the upper atmosphere. Among other things, these phenomena may provide new clues to understand the physics of a lightning bolt.

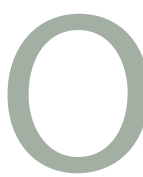

$\mathrm{n}$ the night of July 5-6, 1989 a group of researchers led by the late John $\mathrm{R}$. Winckler from the University of Minnesota was testing a low-light-level camera to be used in a sounding rocket. They pointed the camera northwards to a clear star field that happened to be just above a far active thunderstorm on the horizon. Two frames from their recording [1] showed a couple of huge flashes, extending tens of kilometers above the thunderstorm. What they had serendipitously observed was later called "sprites" by D. Sentman at the University of Alaska, Fairbanks, inspired by W. Shakespeare's “A Midsummer Night's Dream." Two decades after their discovery, sprites are providing clues about the Earth's mesosphere that they inhabit, and they help scientists to understand the nature of lightning discharges. Today we also know that sprites are just one species among a zoo of Transient Luminous Events (TLEs) in the upper atmosphere (see Figure 1).
$\Delta$ the beginning of the storm over the lake CistockPhoto 


\section{The three stages of lightning}

A lightning discharge develops in three stages [2] first, the collisions between ice particles and water droplets together with gravity separate positive and negative charges inside the thundercloud and build up a voltage. Then a discharge grows, forming a socalled streamer-leader tree. Finally, when a conducting path is created between cloud and ground, the cloud discharges through the so-called return stroke; currents then reach tens or hundreds of $\mathrm{kA}$, and the released Ohmic heat brings the channel temperatures up to $28000 \mathrm{~K}$.

Today some of the hottest topics in lightning research are related to the second stage: the inception, growth and branching of a conducting channel in non-ionized air. Under normal circumstances, air is a quite poor conductor because free electrons rapidly attach to oxygen molecules. However, in a sufficiently strong electric field (higher than $3.2 \mathrm{MV} / \mathrm{m}$ in air at standard temperature and pressure) a free electron can gain enough energy to liberate a second electron when it impacts on a molecule, and an ionization avalanche sets in.

However, in most electrical discharges, including lightning, the background electric field is almost everywhere one to three orders of magnitude below the breakdown value. Therefore a simple (linear) ionization avalanche will not grow. The basic breakdown mechanism under these circumstances is provided by so-called "streamers": a sort of adventurers that delve into non-ionized air and pave the way for further ionization waves. They are thin, ionized channels embedded in non-ionized air in an electric field below the breakdown value of $3.2 \mathrm{MV} / \mathrm{m}$ (see figure 2). The ionized region acts as a conductor and enhances the field around its narrow tip, focusing it into a small area where it can reach several times the breakdown value. By ionizing the air in this area, and by moving the space charge layer forward, the streamer advances and focuses the field into a region further ahead. The streamer extends with a velocity of $10^{5}$ to $10^{7} \mathrm{~m} / \mathrm{s}$ that relates to the drift velocity of free electrons.

In a lightning discharge, a corona of streamers paves the way for the so-called "leader". While streamers essentially stay cold and eventually return to their non-conducting equilibrium state, leaders maintain conductivity through higher currents and consecutive Ohmic heating and eventually support the short-circuit between cloud and ground.

Streamers therefore are an important but elusive process in lightning discharges, but they occur in splendid isolation in two other cases. First, they very efficiently convert pulsed electric power into chemical products like ozone that can be used for disinfection, air cleaning or break-up of volatile organic components;

V FIG. 1: The zoo of transient luminous events (TLE's) generated by thunderstorms. Most names are taken from Shakespeare's Midsummer Night's Dream. Elves and sprites are the most frequent. Ground and ionosphere can be considered as equipotential; the thundercloud is the voltage supply separating electrical charges. The break-down electric field is proportional to air density; therefore it decreases strongly with altitude. [Fig. by D.D. Sentman, Univ. Alaska in Fairbanks.]

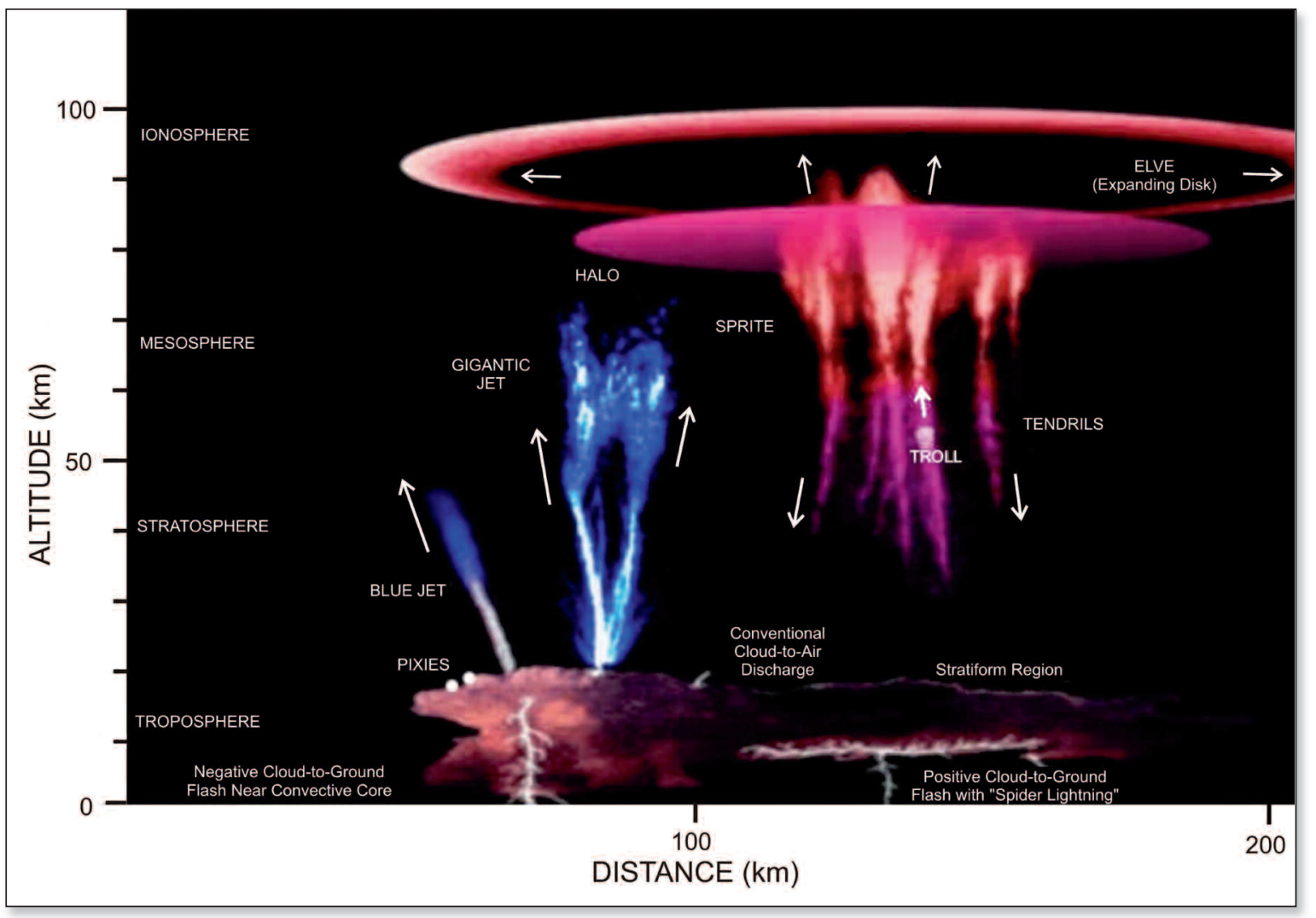


industrial applications are very energy efficient, if the subsequent plasma heating is avoided [3]. Second, streamers appear in a most spectacular manner above thunderstorms in the form of sprite discharges, as first recorded by Winckler and his team.

\section{Firework above the thunderclouds}

Although the first scientifically documented observation of sprites in 1989 was by accident, it was not completely unexpected. In 1925, C.T.R. Wilson, who later won a Nobel Prize for his invention of the cloud chamber, had already suggested a mechanism for the initiation of discharges above a thundercloud [4].

The electrical breakdown threshold depends on air density. The reason is that electrons can gain energy more easily from a given electric field if they experience fewer collisions. If we move upwards in the atmosphere, the air density decreases exponentially (by about a factor 2 every $5 \mathrm{~km}$ ) and so does the breakdown field. But the electric field created by the return stroke together with the equipotential earth is approximately dipolar and hence decays more slowly. Therefore above a certain altitude the breakdown threshold is reached and a discharge is initiated: this is the basic mechanism of sprites. Sprites are composed of streamers, the same structures that pave the way for lightning, only rescaled by the lower air density to be tens to hundreds of meters wide and tens of kilometers long (see Box about scaling). This has been confirmed by high-speed recordings of sprites at up to 10000 frames per second [5] and laboratory photographs with exposure times of nanoseconds [6]. In both cases one sees that light is emitted predominantly from a small region around the tip of the channel, where the electric field is high and ionization is significant.

There are several reasons why sprites have become a hot research topic among physicists and geoscientists. First, they allow us to study the physical process that guides the propagation of a thunderbolt, but without the added complication of streamer-leader transition and return stroke. Leaders are probably not present in upper atmospheric discharges because Ohmic heating is much weaker there; this follows from evaluating scaling laws (see Box) for the Poisson equation [7]. So if you want to understand a lightning stroke, you better might look tens of kilometers above it.

Second, sprites inhabit one of the less known layers of the Earth's atmosphere: the mesosphere; sometimes also termed the "ignorosphere". At those altitudes, air is too rarefied to support a balloon but still would cause too much friction to a satellite; therefore there are very few direct measurements of air properties and chemistry. Sprites generate intense optical emissions and a careful study may reveal more information about the mesosphere.

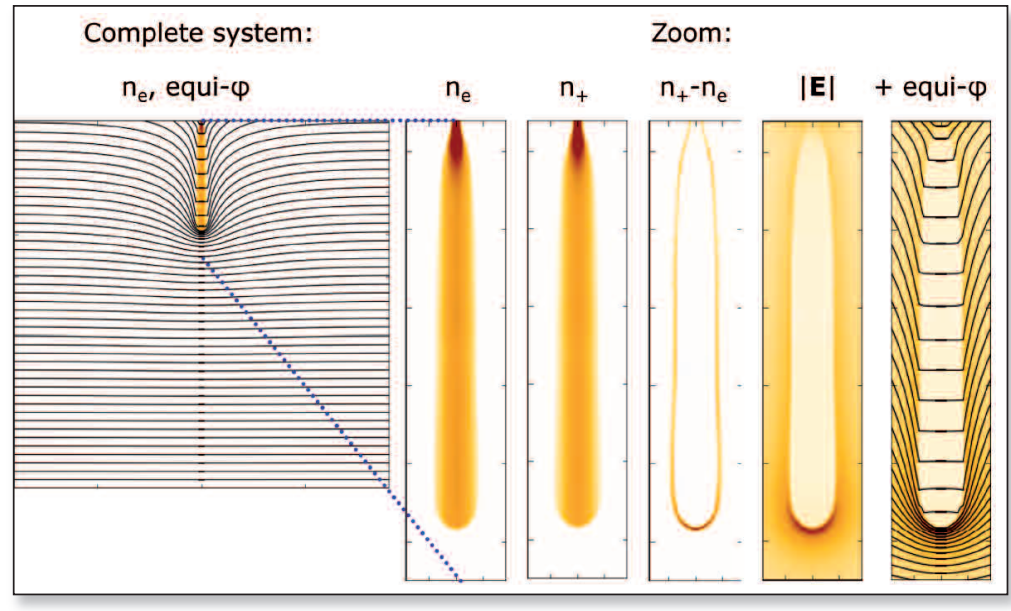

$\Delta$ FIG. 2: Simulation of a streamer discharge in air between two planar electrodes. The streamer starts from a needle electrode inserted into the upper electrode. Shown are from left to right: the complete simulation volume below the needle electrode with electron density in the streamer (colored) and electrical equipotential lines; and zooms into the streamer finger with electron density $n_{e}$, density of positive ions $n_{+}$, space charge density $n_{+}-n_{e}$, electric field strength $|E|$, and the field strength again, now with equipotential lines overlaid. [Fig. by V. Ratushnaya, CWI Amsterdam.]

\section{Observing and modeling sprites}

Since sprites were first observed by Winckler's team, researchers have steadily improved their instruments. Now it is possible to obtain time-resolved movies of the complete development of a sprite [5], [8] (see figure 3 ) and perform detailed spectroscopy of the emitted light.

To remove the atmospheric absorption and scattering, several teams have decided to move their instruments to space. This approach was pioneered by the joint USA-Taiwan instrument ISUAL that has been operating aboard the FORMOSAT-2 satellite since 2004. Europe will have its own orbiting instruments soon: the European Space Agency (ESA) is now building an instrument called ASIM (Atmospheric Space Interactions

\section{Scaling laws}

The dominant length scale in a typical streamer discharge is the electron mean free path / between ionizing collisions with neutral molecules. It is inversely proportional to the molecule density $n$ of the gas: $/ \sim 1 / n$. Therefore all lengths determined by electron motion scale like $1 / n$. The kinetic energies of the electrons have to reach the ionization threshold of the gas molecules which is a molecule-specific value and independent of the gas density; therefore the characteristic electron energies do not depend on gas density. Therefore also the characteristic electron velocities are independent of gas density $n$. As lengths scale like $1 / n$ and electron velocities are independent of $n$, times have to scale as $1 / n$ as well. As energies are independent of $n$, voltages are independent of $n$, and therefore electric fields scale as $n$, as they have dimension of voltage over length.

The air density at $83 \mathrm{~km}$ above sea level is about 100.000 times smaller than at ground level. Therefore if lengths and times are measured in centimeters and nanoseconds for an atmospheric pressure streamer, they scale to kilometers and fractions of milliseconds in a sprite at $83 \mathrm{~km}$. 


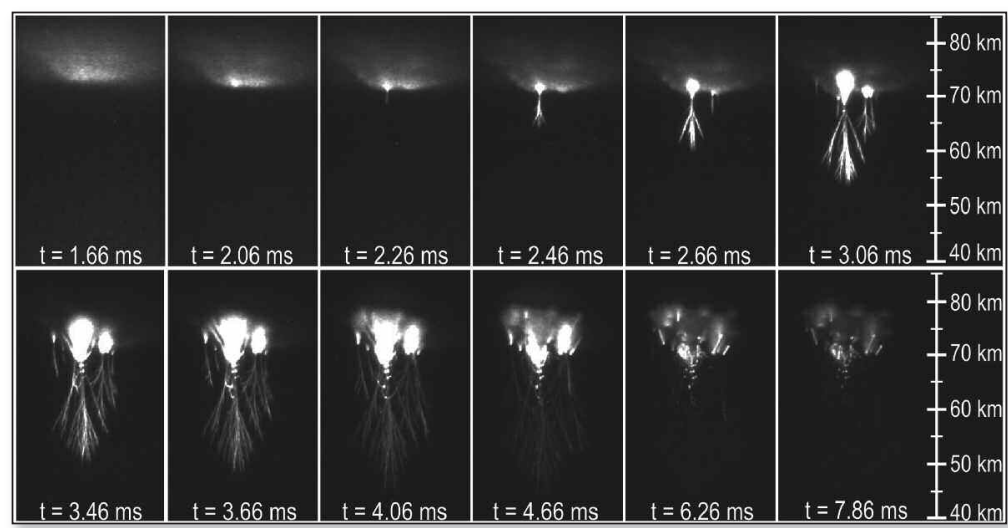

$\Delta$ FIG. 3: The evolution of a halo up to time $t=2.06 \mathrm{~ms}$ and the successive sprite discharge above a thundercloud is filmed with $\mathbf{5 0 0 0}$ frames per second. Atmospheric altitude is indicated on the right. At time $t=0$, a lightning return stroke starts transporting electrical charge to earth, generating an electric field above the cloud. This field creates first a halo at the lower edge of the ionosphere and then a sprite. In the lower row, one can also see the reconnection process between a streamer tip and a pre-existing channel [8].

III Monitor) that will operate from the International Space Station; its launch is scheduled for 2013. Also the French CNES develops their own micro-satellite TARANIS (Tool for the Analysis of RAdiation from lightNIng and Sprites), scheduled for 2015.

Given the complexity and inaccessibility of the sprite phenomena, it is not surprising that computer simulations are playing an increasingly important role.

One example is the initiation of sprites from halos. Halos are wide, saucer-shaped diffuse optical emissions from the lower ionospheric edge that often precede the sprite (see figure 3). Using realistic altitude and ionization profiles, our simulations [9] could follow that process and show how after a lightning discharge the boundary of the ionosphere is sharpened into an unstable edge, from which a streamer shoots out (figure 4).

Also with the aid of simulations, we have postulated that the upper portions of a sprite streamer channel are negatively charged [10]. That may explain why frequently the positively charged streamer tips are attracted to a previous channel, as seen also in figure 3 .

V FIG. 4: Simulation of halo and emerging sprite. The simulation uses a gas discharge model, air density varying with altitude, an initial electron density profile corresponding to fair weather night time conditions and the electric field generated by lightning stroke and ionospheric response. The largely varying length scales (down to scales of meters in the space charge layer of the sprite) are caught through adaptively refined numerical grids moving with the structure [9].

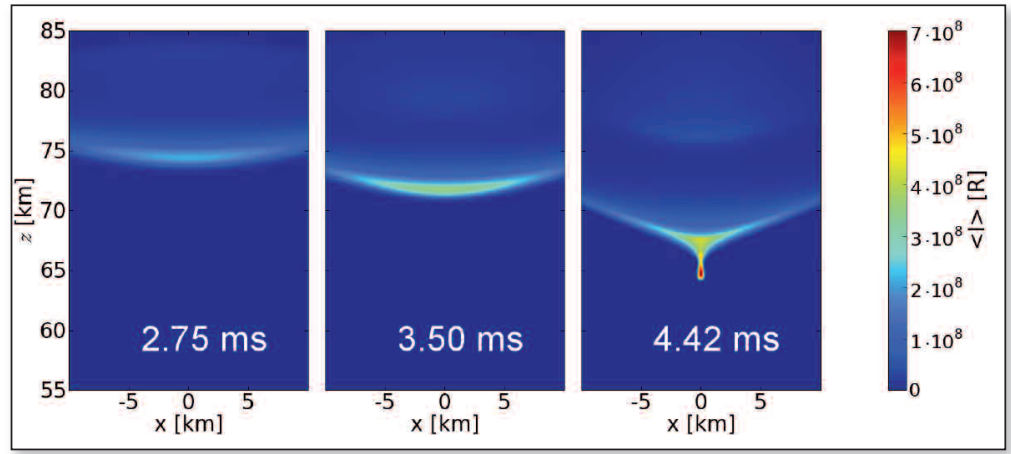

\section{Outlook}

In the 20 years since their first observation, research has established some basic characterization and understanding of sprites. But many questions remain: What does the emergence altitude of sprites tell us about the state of the meso- and ionosphere? How many greenhouse gases are produced by lightning and sprites, and in which stage of the discharge does this happen? Are terrestrial gamma-ray flashes (TGFs) related to sprites? TGFs [11] are sudden bursts of highly energetic radiation associated with lightning strokes whose precise origin is under debate. Do sprites exist on other planets [12]? There they would be the first observable signature of lightning when approaching the planet from space.

Lightning and upper-atmospheric discharges are surely among the most spectacular phenomena in nature. And physically similar streamer discharges are most interesting in plasma and high voltage technology and in plasma medicine. The same research therefore can create new technology, explain lightning, and explore our mesosphere and the atmospheres of other planets from a distance.

\section{About the authors}

A. Luque studied physics in Sevilla (Spain), obtained his $\mathrm{PhD}$ in Bayreuth (Germany) and was a postdoc in Bochum (Germany) and at CWI Amsterdam (The Netherlands). He is currently at the Institute of Astrophysics of Andalusia (IAA-CSIC, Spain).

U. Ebert studied physics in Heidelberg (Germany), obtained her PhD in Essen (Germany), was postdoc in Leiden (The Netherlands) and moved to CWI Amsterdam in 1998. She now leads a research group at CWI and is parttime professor of physics in Eindhoven (The Netherlands)

\section{References}

[1] R.C. Franz, R.J. Nemzek, J.R. Winckler, Science 249, 48 (1990).

[2] For an exhaustive review of lightning phenomena, see e.g. V. Rakov, and M. Uman, Lightning: Physics and Effects, Cambridge University Press, Cambridge, U. K. (2003).

[3] E.J.M. van Heesch, G.J.J. Winands, and A.J.M. Pemen, J. Phys. D: Appl. Phys. 41, 234015 (2008).

[4] C. T. R. Wilson, Proc. Phys. Soc. London 37, 32D (1925).

[5] H.C. Stenbaek-Nielsen, and M.G. McHarg, J. Phys. D: Appl. Phys. 41, 234009 (2008)

[6] T.M.P. Briels, E.M. van Veldhuizen, and U. Ebert, J. Phys. D: Appl. Phys. 41, 234008 (2008).

[7] U. Ebert, S. Nijdam, C. Li, A. Luque, T. Briels, and E. van Veldhuizen, J. Geophys. Res. 115, A00E43 (2010). doi:10.1029/2009JA014867.

[8] S.A. Cummer, N. Jaugey, J. Li, W.A. Lyons, T.E. Nelson, and E.A. Gerken, Geophys. Res. Lett. 33, L04104 (2006).

[9] A. Luque, and U. Ebert, Nature Geoscience 2, 757 (2009).

[10] A. Luque, and U. Ebert, Geophys. Res. Lett. 37, L06806 (2010).

[11] G.J. Fishman et al., Science 264, 1313 (1994).

[12] Y. Yair, Y. Takahashi, R. Yaniv, U. Ebert, Y. Goto, J. Geophys. Res. 114, E09002 (2009) 